\title{
Acute exacerbation of idiopathic pulmonary fibrosis: frequency and clinical features
}

\author{
D.S. Kim*, J.H. Park*, B.K. Park*, J.S. Lee ${ }^{\#}$, A.G. Nicholson` and T. Colby
}

ABSTRACT: Although acute exacerbations of idiopathic pulmonary fibrosis are well recognised, there are no studies documenting their prevalence or identifying pre-existing risk factors.

This study analysed the clinical, radiological and pathological data of 11 patients who satisfied the criteria for acute exacerbation among 147 patients with biopsy-proven idiopathic pulmonary fibrosis. There were five additional patients who had similar demographics, radiology and surgical lung biopsy pathology, but had clinically less severe disease, and so were not included. The 2-yr frequency of acute exacerbation was $9.6 \%$ after the diagnosis. Most exacerbations were idiopathic, although two cases presented after surgical lung biopsy and one after bronchoalveolar lavage.

No significant risk factor was found by univariate proportional hazard analysis. Imaging revealed diffuse bilateral ground-glass opacification superimposed on subpleural reticular and honeycombing densities. The biopsies of four patients taken during acute exacerbation exhibited diffuse alveolar damage superimposed upon usual interstitial pneumonia.

The findings of this study demonstrate that acute exacerbation of idiopathic pulmonary fibrosis is rather common and this exacerbation is likely to have a spectrum of severity.

KEYWORDS: Acute exacerbation, diagnostic criteria, frequency, idiopathic pulmonary fibrosis, risk factors, surgical lung biopsy pathology

I diopathic pulmonary fibrosis (IPF) is a fatal fibrotic lung disease of no identifiable aetiology, characterised by a histological pattern of usual interstitial pneumonia (UIP) [1-5]. Although the clinical course is usually chronic and slowly progressive [1-5], some patients experience rapid deterioration during the course of their illness. Most reports on this phenomenon of acute exacerbation (AE) of IPF have appeared in the Japanese medical literature [6-14]. In the English medical literature, COLBY [15] and KITAICHI [16] first reported the pathological findings of $\mathrm{AE}$ as diffuse alveolar damage (DAD) superimposed on UIP. KONDOH et al. [17] described clinical and pathological data on three patients with $\mathrm{AE}$ and later AKIRA et al. [18] reported radiological data on 17 patients. Recently, RICE et al. [19] reported the autopsy findings of DAD patterns superimposed on UIP in 12 patients with IPF. AMBROSINI et al. [20] also recently described the clinical and pathological features of five cases. However, the clinical features of this phenomenon are still unclear and the definition of $\mathrm{AE}$ varies from author to author regarding both the degree of severity and rapidity.

The clinical features of 11 patients who satisfied the original criteria by $\mathrm{KONDOH}$ et al. [17] have since been reviewed at the authors' Interstitial Lung Disease Clinic (Asan Medical Center, Seoul, Korea); four of the 11 underwent surgical lung biopsies (SLBx) at the time of AE.

\section{SUBJECTS AND METHODS \\ Study design and subjects}

This is a retrospective descriptive study. In total, 147 patients were reviewed; they had been diagnosed as IPF by SLBx according to the American Thoracic Society (ATS)/European Respiratory Society (ERS) Consensus Classification [21] from January 1990 to September 2003 at the Asan Medical Center, a 2,000-bed university-affiliated tertiary referral centre in Seoul, Korea. Of these 147 patients, 11 cases satisfied the criteria of AE proposed by $\mathrm{KONDOH}$ et al. [17]. All the patients were diagnosed and followed up by one doctor at the IPF clinic at the Asan Medical Center. Most of the subjects with IPF have been previously reviewed in a study of lung physiology [22].

\section{Criteria of acute exacerbation}

The strict criteria of KONDOH et al. [17] were used to define an event of AE. These included: 1) aggravation of dyspnoea within 1 month; 2) hypoxaemia with a arterial oxygen tension/ inspired oxygen tension ratio of $<225$; 3) newly
AFFILIATIONS

*Dept of Pulmonary and Critical Care Medicine, and

\#Dept of Radiology, Asan Medical Center, Ulsan University, Seoul, South Korea.

'Dept of Pathology, Royal Brompton Hospital, London, UK.

${ }^{+}$Dept of Laboratory Medicine and Pathology, Mayo Clinic, Scottsdale, Arizona, USA.

\section{CORRESPONDENCE}

D.S. Kim

Dept of Pulmonary and Critical Care Medicine

Asan Medical Center

Ulsan University

Poongnap-dong

Songpa-ku

Seoul

Korea

Fax: 82230106968

E-mail: dskim@amc.seoul.kr

Received:

October 022004

Accepted after revision:

September 092005 
developing pulmonary infiltrates on chest radiography; and 4) absence of apparent infection or heart disease.

\section{Methods}

All clinical and laboratory data were collected retrospectively from medical records.

\section{Pulmonary function test}

Spirometry was performed with a Sensor Medic 2100 (Yorba Linda, CA, USA). Diffusion capacity was measured with a Sensor Medic Model Vmax 22, and lung volumes were measured with a Sensor Medic Auto Box 6200.

\section{High-resolution computed tomography imaging}

High-resolution computed tomography (HRCT) was performed on all patients, usually on the day of admission, with a HiSpeed Advantage Scanner (GE Medical Systems, Milwaukee, WI, USA) or a Somatom Plus 4 scanner (Siemens Medical Systems, Erlangen, Germany). The images were analysed at a window level of $-700 \mathrm{HU}$ and a window width of 1,500 HU. One radiologist (J.S. Lee) reviewed the HRCT of the lungs without knowledge of the biopsy results or clinical outcomes. HRCT findings at the time of AE were classified as peripheral, multifocal, or diffuse parenchymal opacities [18].

\section{Bronchoalveolar lavage}

Bronchoalveolar lavage (BAL) was performed as previously reported [23].

\section{Pathological evaluation}

Biopsy slides were reviewed independently by two pathologists (A.G. Nicholson and T. Colby), first, to classify the background histological pattern of chronic interstitial pneumonia (if present in the sample) according to the ATS/ERS consensus classification [21], and, secondly, to confirm the presence of superimposed acute changes in the form of exudative (with hyaline membranes) or organising DAD. Background chronic interstitial pneumonia, when present, was then scored according to a previously described protocol for the extent of fibroblastic foci, interstitial mononuclear inflammation, alveolar macrophage accumulation, and established fibrosis, using a semiquantitative scale of $0-6$ [24]. The final consensus view of the histological diagnosis was then correlated with HRCT and clinical data to provide the final clinicopathological diagnosis.

\section{Statistical analysis}

Data are expressed as mean $\pm \mathrm{SD}$ for continuous variables, and percentages for categorical variables. BAL data are expressed as median values with ranges. Categorical data were compared using a Chi-squared test (Pearson's test or Fisher's exact test). The two groups were compared using the unpaired t-test and the Mann-Whitney U-test. All data between the exacerbation and nonexacerbation groups were compared using the MannWhitney U-test, because the exacerbation group consisted of only eight patients. Three patients who presented at the time of $\mathrm{AE}$ were excluded in this comparison, because their data were obtained at $\mathrm{AE}$, and no pre-AE data were available. The frequency of $\mathrm{AE}$ was obtained by the Kaplan-Meier curve constructed for follow-up of patients at the end (death or lost to follow-up) by censoring AE as the "death variable". The risk factors for AE were analysed by a log-rank test (a univariate proportional hazards method) for the development of $\mathrm{AE}$ as the 'death variable' censoring at the end of follow-up or at death. Because of the small numbers involved in this study $(n=11)$, multivariate analysis using the same proportional hazards model was not performed. In all cases, two-sided tests were used; p-values $<0.05$ were deemed statistically significant.

\section{RESULTS}

\section{Frequency of acute exacerbation}

Among the 147 patients that had been diagnosed as IPF by SLBx, 23 had been admitted due to acute aggravation of the disease without apparent infection during follow-up. Of these, seven patients were excluded because infection could not be completely ruled out. Another five patients did not satisfy all Kondoh's criteria (longer duration and/or less severe hypoxaemia), so that even though four of these cases that were biopsied at the time of exacerbation did exhibit features of DAD superimposed upon UIP, they were not included. Therefore, only the remaining 11 patients who satisfied all the criteria of $\mathrm{AE}$ by $\mathrm{KONDOH}$ et al. [17] were the subjects of this study.

On follow-up of IPF in the survivors, the 1-yr frequency of AE was $8.5 \%$ and the 2 -yr frequency was $9.6 \%$.

\section{Risk factors associated with acute exacerbation}

Precipitating factors of AE were not identified in the majority of patients. Two patients, who had previously been stable, developed AE shortly after SLBx. Another patient developed AE immediately after BAL and an SLBx was performed 12 days later, which revealed an organising DAD pattern. Cultures of BAL fluid from this patient and lung biopsy were negative.

When the initial demographic and clinical features of the patients with $\mathrm{AE}$ were compared with the patients without $\mathrm{AE}$ using a univariate proportional hazard method, no significant risk factors were found (table 1).

\section{Clinical features of the patients with acute exacerbation}

The clinical features of the subjects with AE are summarised in table 2 . Among the 11 patients who satisfied Kondoh's criteria, five developed AE 13.3 \pm 11.7 months (range: 3-60 months) after the diagnosis of IPF by surgical lung biopsy. Three of the 11 developed AE immediately after diagnostic procedures (one after BAL and two after SLBx). The remaining three patients presented with AE with no prior diagnosis of IPF, however, two had exhibited dyspnoea for 6 and 2 months but had not visited the hospital until the symptoms became acutely worse at 20 and 14 days before admission, respectively. The remaining patient had noticed dyspnoea 1 month before admission, which had progressed rapidly. Surgical lung biopsies were performed at the time of $\mathrm{AE}$ in four patients (three patients who presented with $\mathrm{AE}$ and one patient who developed $\mathrm{AE}$ after $\mathrm{BAL}$ ). The average time from $\mathrm{AE}$ onset to admission was $13.0 \pm 10.7$ days (range: $2-30$ days). A rapidly progressive dyspnoea $(100 \%)$ was the most prominent symptom, with associated cough (55\%) and scanty sputum (46\%). A mild fever was present in one patient and none of the patients had evidence of heart disease. 
TABLE 1 Comparison of clinical features between subjects with and without acute exacerbation

\begin{tabular}{|c|c|c|c|c|c|}
\hline & $\begin{array}{l}\text { Acute exacerbation by Kondoh's } \\
\text { criteria }\end{array}$ & Nonexacerbation & p-value ${ }^{\#}$ & Accelerated decline & p-value \\
\hline Age yrs & $63.4 \pm 6.3$ & $58.6 \pm 9.6$ & NS & $60.1 \pm 9.4$ & NS \\
\hline Sex $M / F$ & $6: 2$ & $92: 32$ & NS & $11: 5$ & NS \\
\hline \multicolumn{6}{|l|}{ Smoking } \\
\hline Dyspnoea MRC & $2.7 \pm 1.1$ & $2.8 \pm 1.4$ & NS & $2.7 \pm 1.0$ & \\
\hline \multicolumn{6}{|l|}{ PFT" \% pred } \\
\hline FVC & $72.5 \pm 13.5$ & $73.3 \pm 17.7$ & NS & $67.8 \pm 16.5$ & NS \\
\hline $\mathrm{DL}, \mathrm{CO}$ & $54.0 \pm 23.1$ & $64.1 \pm 19.2$ & NS & $56.4 \pm 21.4$ & NS \\
\hline TLC & $84.1 \pm 18.5$ & $78.8 \pm 17.6$ & NS & $75.1 \pm 21.8$ & NS \\
\hline Neutrophils \% & $4.0(3.0-35.6)$ & $6.5(0.0-27.7)$ & NS & $7.0(0.6-41.0)$ & NS \\
\hline Eosinophils \% & $5.0(0.0-15.0)$ & $0.6(0.0-22.0)$ & NS & $3.0(0.0-15.0)$ & NS \\
\hline Lymphocytosis" $">20 \%$ & $5: 7(71.4 \%)$ & $28: 96$ (29.2\%) & NS & $6: 16(40.0 \%)$ & NS \\
\hline Neutrophilia" > $5 \%$ & $3: 7(42.9 \%)$ & $59: 96(61.5 \%)$ & NS & $9: 16(60.0 \%)$ & NS \\
\hline Eosinphilia >1.5 \% & $4: 7(57.1 \%)$ & $39: 92(42.4 \%)$ & NS & $10: 15(66.7 \%)$ & NS \\
\hline
\end{tabular}

Data presented as $\mathrm{n}$, ratio, mean $\pm \mathrm{SD}$ or median (range). M: male; F: female; MRC: (British) Medical Research Council; PFT: pulmonary function test; FVC: forced vital capacity; DL,CO: carbon monoxide diffusing capacity of the lung; TLC: total lung capacity; $\mathrm{Pa}_{\mathrm{a}} \mathrm{O}_{2}$ : arterial oxygen tension; BAL: bronchoalveolar lavage; NS: nonsignificant. ${ }^{\#}$ : $\mathrm{p}$-value between patients with and patients without acute exacerbation (AE); ${ }^{\top}: \mathrm{p}$-value between patients with accelerated decline and patients without $\mathrm{AE} ;{ }^{+}$: among 11 patients with $\mathrm{AE}$, three patients who presented at the time of $\mathrm{AE}$ were excluded because no pre-AE data were available; ${ }^{\varsigma}$ : the number of patients with idiopathic pulmonary fibrosis after exclusion of "accelerated decline", which includes definite AE (11 patients), suspicious AE but not satisfying the Kondoh's criteria (five patients) and diagnostic uncertainty (seven patients); ${ }^{\text {: }}$ among 23 patients with "accelerated decline", seven patients who presented at the time of AE were excluded because no pre-AE data were available; $\# \#$ : patients treated with steroids either with or without cytotoxic drugs. In the AE group, this is the number of patients treated before AE. In the non-AE group, this is the number of the patients treated during the course of the disease; $" \%$ : at the time of diagnosis.

\section{Bronchoalveolar lavage findings}

Eight of 11 patients underwent BAL at the time of $\mathrm{AE}$; the remaining three developed AE after BAL and SLBx. Three patients had only elevated neutrophils $(>5 \%)$ and five patients had increases in both neutrophils and lymphocytes $(>20 \%)$ (table 2).

\section{Microbiological studies}

All cultures of blood, sputum, and urine collected at the time of $\mathrm{AE}$ were negative. Cultures of BAL fluid performed at the time of $\mathrm{AE}$ in eight patients, excluding those with procedurerelated $\mathrm{AE}$, were all negative for bacteria, fungi, and viruses, as were tests for Pneumocystis carinii and cytomegalovirus. The lung tissues obtained by SLBx at the time of AE were all cultured, with no organisms grown. Serological tests for mycoplasma, Legionella, and viruses were undertaken in 10 patients (the patient who developed AE after the BAL was not tested) and all were negative. Cultures of blood from one patient and sputum from one patient, both samples collected during mechanical ventilation in the course of the illness, were positive for aerobic bacteria. These were thought to represent either contamination or were the cause of ventilator-associated pneumonia.

\section{Radiological features of acute exacerbation}

High-resolution computed tomography findings

All eight patients displayed newly developed diffuse bilateral ground-glass opacity (GGO) at the time of $\mathrm{AE}$, superimposed on subpleural reticular and honeycombing densities (figs. 1-3). The HRCT of three patients who presented with AE without prior diagnosis also exhibited bilateral honeycombing in addition to diffuse GGO. The distribution pattern of new GGO was primarily peripheral in three patients, multifocal in seven, and diffuse in one (table 3).

\section{Pathological findings of acute exacerbation}

Seven patients had received SLBx prior to AE and two of the seven developed AE immediately after SLBx. The pathological consensus diagnosis for these seven patients was UIP without acute changes. The remaining four patients had SLBx at the time of AE and all cases exhibited UIP with superimposed $\mathrm{DAD}$. The DAD was predominantly in the organising phase in two of the cases with interstitial and airspace organisation, interstitial oedema, and type 2 cell hyperplasia. More acute DAD with hyaline membranes was the dominant finding (relative to the organisation) in only two patients (fig. $4 \mathrm{~b}$ ), and hyaline membranes were found in one of the other two cases 


\begin{tabular}{|c|c|c|c|}
\hline \multirow[t]{2}{*}{ TABLE 2} & \multicolumn{3}{|c|}{$\begin{array}{l}\text { Clinical and laboratory findings at the time of } \\
\text { acute exacerbation ( } A E \text {; by Kondoh's criteria) }\end{array}$} \\
\hline & & Mean \pm SD & Range \\
\hline \multicolumn{2}{|c|}{ Duration from IPF diagnosis months } & $13.3 \pm 11.7$ & $3-60$ \\
\hline \multicolumn{2}{|c|}{$\begin{array}{l}\text { Duration of acute symptom before } \\
\text { admission days }\end{array}$} & $13.0 \pm 10.7$ & $2-30$ \\
\hline \multicolumn{2}{|c|}{ Fever $\mathbf{n}$} & 1 & \\
\hline \multicolumn{2}{|l|}{ 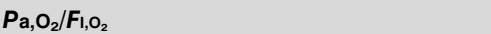 } & $183 \pm 26.0$ & $99-227$ \\
\hline \multicolumn{4}{|c|}{ Bronchoalveolar lavage fluid finding } \\
\hline \multicolumn{2}{|c|}{ Macrophages \% } & $45.6 \pm 23.9$ & $13-90$ \\
\hline \multicolumn{2}{|c|}{ Lymphocytes \% } & $28.6 \pm 18.5$ & $3-48$ \\
\hline \multicolumn{2}{|c|}{ Neutrophils \% } & $24.7 \pm 16.9$ & $5-48$ \\
\hline \multicolumn{2}{|c|}{ Eosinophils \% } & $2.7 \pm 3.6$ & $1-15$ \\
\hline \multicolumn{2}{|c|}{ WBC $\times 10^{3} \cdot \mathrm{mm}^{-3}$} & $12.2 \pm 3.7$ & $6.6-17.8$ \\
\hline \multicolumn{2}{|c|}{ C-reactive protein } & $12.6 \pm 6.0$ & $3.5-20.3$ \\
\hline \multicolumn{2}{|c|}{ Corticosteroid pulse therapy $n$} & 6 & \\
\hline \multicolumn{2}{|c|}{ ICU admission $n$} & 10 & \\
\hline \multicolumn{2}{|c|}{ Mechanical ventilation $n$} & 9 & \\
\hline \multicolumn{2}{|c|}{ Mortality within 3 months $\mathrm{n}$} & 9 & \\
\hline \multicolumn{2}{|c|}{ Duration between the $A E$ and death days } & $33.2 \pm 21.4$ & $11-70$ \\
\hline
\end{tabular}

with a dominant pattern of intra-alveolar organisation. Whilst on a field-per-field basis, the features may be identical to organising pneumonia, as seen in cryptogenic organising pneumonia (COP) (fig. 5b), the presence of the hyaline membrane plus the clinical context indicate the appropriate classification as the organising phase of DAD. The airspace organisation in these cases was also generally distinguishable from the fibroblastic foci of UIP seen at the junction between intact and scarred lung.

\section{Clinical courses of acute exacerbation}

All patients were treated with broad-spectrum antibiotics and corticosteroids, and six received corticosteroid pulse therapy (methylprednisolone $1 \mathrm{gm} \cdot \mathrm{day}^{-1}$ for 3 days).

Among nine patients who were mechanically ventilated, the hospital mortality rate was $78 \%$. Four patients survived the initial episodes, but two of these died within 3 months (the overall mortality rate was $81.8 \%$ ). The cause of death was progressive disease, except in one patient (ventilatorassociated pneumonia). Among the three patients who developed procedure (BAL and SLBx)-related AE, only one (after SLBx) died. The HRCT pattern at the time of AE had significant prognostic value $(p=0.042)$; all three patients with the peripheral type of GGO and one with the multifocal type survived their initial episodes (table 3). There were two cases of recurrence of $\mathrm{AE}$, which developed similar exacerbations 2.5 months and 9 months later and died at those times.

\section{DISCUSSION}

In the authors' experience with a large series of patients with IPF, the 1-yr frequency of AE in IPF was $8.5 \%$ and the 2-yr frequency was $9.6 \%$. However, the actual incidence in this study could be even higher as: 1) some patients were lost

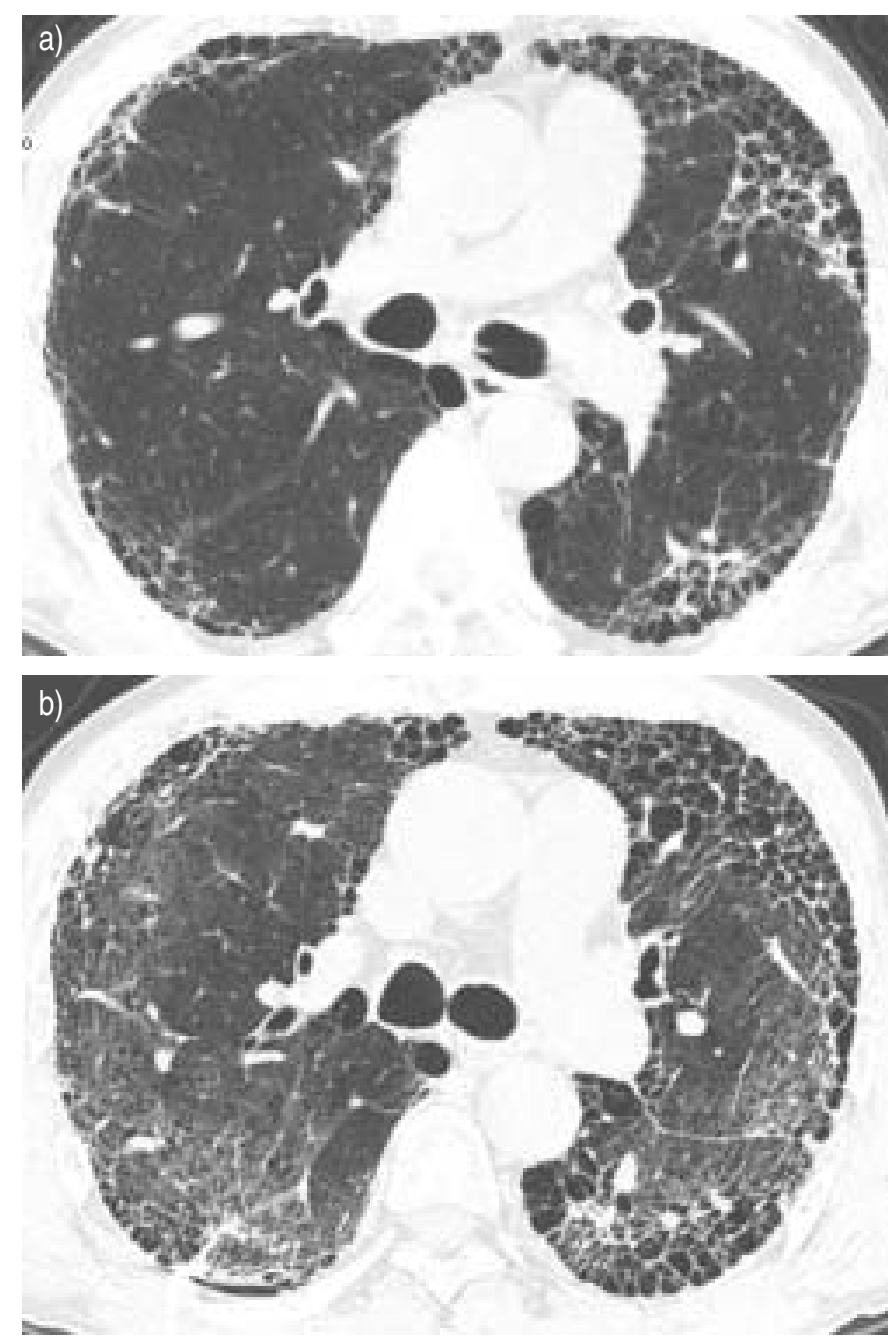

FIGURE 1. A 63-yr-old male with idiopathic pulmonary fibrosis and acute exacerbation showing peripheral type. a) Initial high-resolution computed tomography scan shows the reticular opacity and severe honeycombing in the subpleural area of both lungs. b) On follow-up computed tomography after 2 months, the ground-glass opacities of peripheral subpleural distribution have developed bilaterally.

during follow-up; 2) even among the hospitalised patients, not all the patients with acute aggravation were included in this study due to diagnostic uncertainty; 3 ) only those with surgical confirmation of UIP were included; and 4) another five patients who did not strictly satisfy all of Kondoh's criteria and so were also excluded, did in fact have the clinical and surgical lung biopsy pathology features (of four among them), strongly suggesting that they were cases of AE. A univariate proportional hazards analysis of demographic features and lung function parameters between the patients with $\mathrm{AE}$ and the patients who did not develop AE failed to identify significant risk factors.

IPF is typically a chronic disease with a mean/median survival time of $\sim 3$ yrs $[1,3,4]$, although the rate of progression of the disease varies [25]. Acute deterioration may occur secondary to infections, pulmonary embolism, pneumothorax, or heart failure [26], but it may also occur without an identifiable 


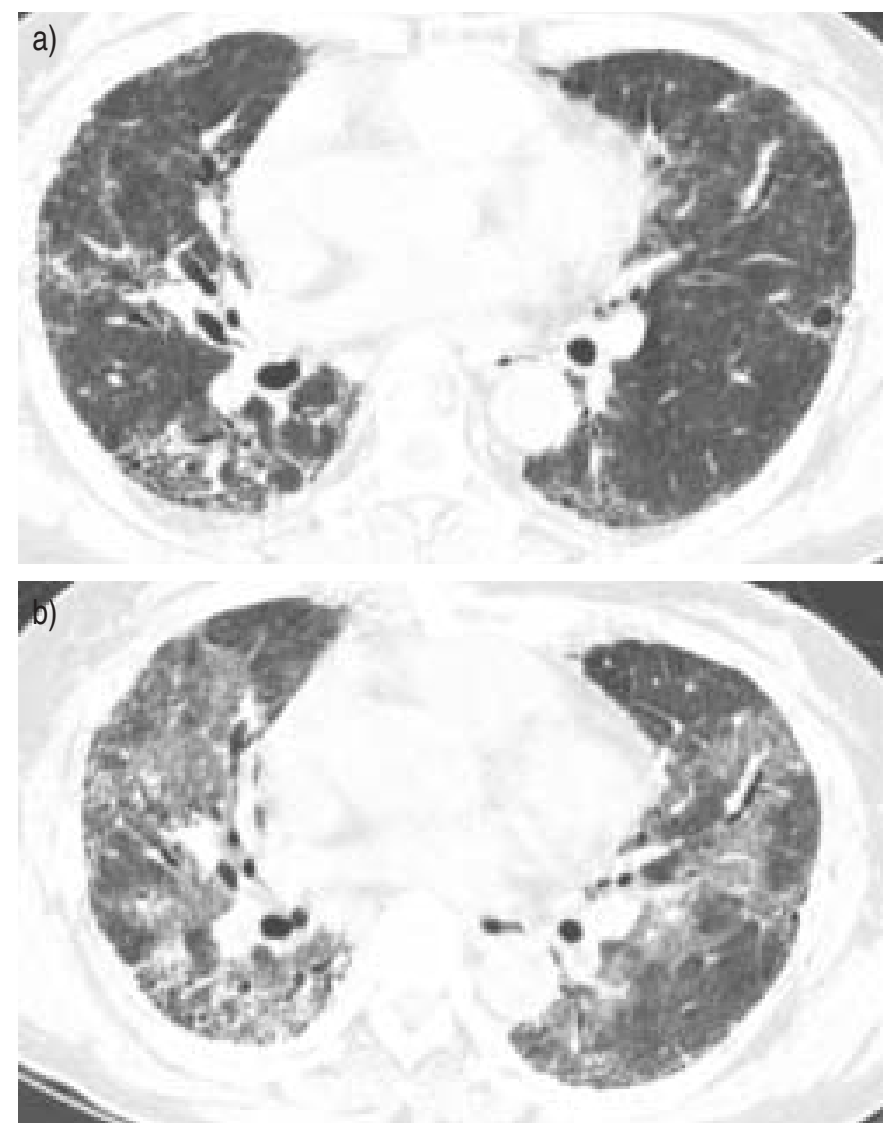

FIGURE 2. A 57-yr-old female with idiopathic pulmonary fibrosis and acute exacerbation showing multifocal patchy type. a) An initial high-resolution computed tomography scan shows the reticular opacity and honeycombing in the subpleural area of the lung. b) In follow-up computed tomography after 1 month, the groundglass opacities of multifocal patchy distribution have developed in both lungs. The patchy ground-glass opacities are distributed randomly and the fine reticular opacities can also be seen within the ground-glass opacity.

cause, which is termed AE of IPF [6-20, 27, 28]. However, the clinical features of this phenomenon are not clear and there is no widely accepted definition of AE. The definition of AE has varied somewhat in terms of severity and rapidity from author to author. According to KONDOH et al. [17], the criterion of hypoxaemia is a oxygen tension/inspired oxygen tension ratio of $<225$, while AKIRA et al. [18] uses the criterion of $>1.33 \mathrm{kPa}$ $(10 \mathrm{mmHg})$ reduction under similar conditions. Furthermore, even though the duration of aggravating dyspnoea is defined as $<1$ month (or a few weeks) in most definitions, the time from the onset of rapid deterioration before admission of the subjects of RICE et al. [19] was 3-60 days and that of AKIRA et al. [18] was 2-44 days. This study included only subjects satisfying the strictest criteria of $\mathrm{KONDOH}$ et al. [17] in order to concentrate on the data in these core patients, which led to the exclusion of five patients who satisfied the less strict criteria of AKIRA et al. [18]. Four of these patients had similar histopathological findings to those in the studied cohort, suggesting that there may be a range of disease progression in IPF from the typical chronically progressive disease to the overt AE as defined by KONDOH et al. [17]. Kondoh's criteria were important because they represent the first attempt to

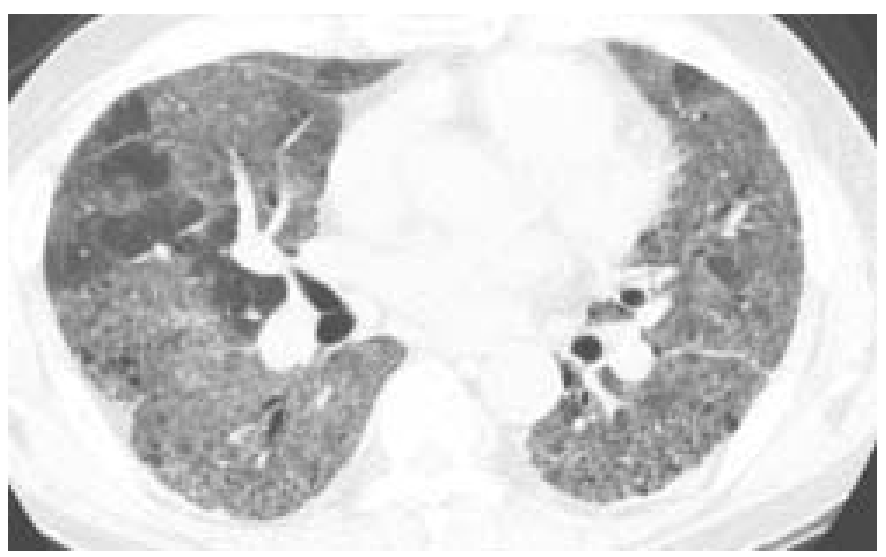

FIGURE 3. A 56-yr-old male with idiopathic pulmonary fibrosis and acute exacerbation showing diffuse type. The patient first presented to the Asan Medical Center at the time of acute exacerbation and high-resolution computed tomography shows diffuse ground glass opacities on both lungs. On close observation, honeycombing and some reticular densities can be seen in the subpleural area of both lower lungs within the ground glass densities. The patient expired 22 days after the admission.

define a previously unknown entity; however, this study showed that it should be redefined. When baseline disease severity was mild before the onset of $\mathrm{AE}$, the same severity of accelerated decline might not result in sufficiently severe hypoxia to satisfy Kondoh's criteria. Therefore, in this respect, the criteria of AKIRA et al. [18] seemed to be more appropriate. Also, the onset of aggravation of dyspnoea may be extended to several weeks. If Akira's criteria had been used and the seven patients in whom the infection could not be completely ruled out had also been included, the 1-yr frequency would be $15.4 \%$ with a 2 -yr frequency of $18.0 \%$. When those patients with accelerated decline were compared with the patients with $\mathrm{AE}$ (as defined by Kondoh), no significant difference in any of the parameters of acute exacerbation (table 2) was found between the two groups; also, no significant risk factors for the development of "accelerated decline" were found.

Even though it was believed that all patients should have preexisting diagnosis of IPF and manifest AE during follow-up, three patients did not have an established diagnosis of IPF before the manifestation of AE. Not only the presence of dyspnoea before the presentation to the hospital in two of them, but also the presence of a significant amount of honeycombing on HRCT of all three patients in addition to diffuse GGO strongly suggests underlying pre-existing IPF. In addition, the presence of pathological findings of UIP, such as subpleural microscopic honeycombing, dense fibrosis and fibroblastic foci (not Masson body of bronchiolitis obliterans organising pneumonia pattern) in addition to DAD features points to the diagnosis of AE of IPF rather than AIP. The three patients in this study with definite $\mathrm{AE}$ and also four patients with suspicious AE had all these features on HRCT and pathology.

In keeping with published reports, the aetiology of $\mathrm{AE}$ was unknown in most of the subjects in this study; however, three patients had developed AE immediately after iatrogenic procedures (two after SLBx and one after BAL), and there 
TABLE 3 High-resolution computed tomography patterns at the time of acute exacerbation (number of patients)

CT pattern of GGO

Patients n

\begin{tabular}{lcccc}
\cline { 2 - 4 } & Acute exacerbation $^{\#}$ & Survival from acute exacerbation & Accelerated decline & Survival of accelerated decline \\
\hline Diffuse & 1 & 0 & 3 & 0 \\
Multifocal & 7 & 0 & 14 & 5 \\
Peripheral & 3 & 2 & 6 & 5 \\
Total & 11 & 2 & 23 & 10 \\
\hline
\end{tabular}

All patients had reticular and honeycombing densities in both lower lobes in addition to ground glass density. CT: computed tomography; GGO: ground glass opacity. ${ }^{\text {\# }}$ patients who satisfied the acute exacerbation according to Kondoh's criteria; ". patients with definite acute exacerbation (11 patients), suspicious acute exacerbation but not satisfying Kondoh's criteria (five patients) and diagnostic uncertainty (seven patients).
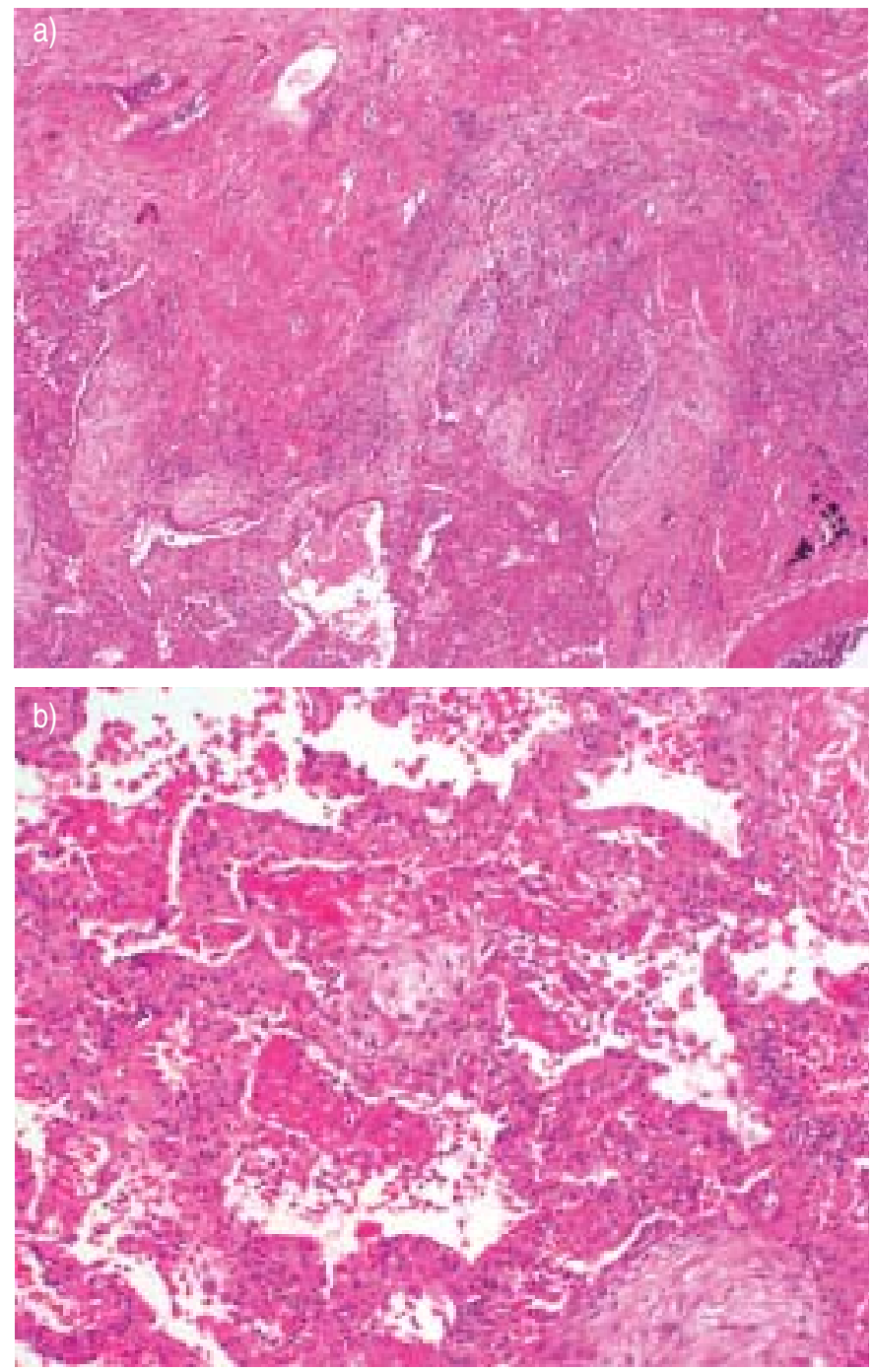

FIGURE 4. A 66 -yr-old male presented at the time of acute exacerbation without prior diagnosis. a) shows an area of dense scarring with paler fibroblast foci (lower half of the figure) adjacent to the more densely eosinophilic older collagen (upper half). b) The region shown exhibits intact lung tissue with acute lung injury manifesting as airspace organisation (centre and lower right) and hyaline membrane and fibrinous exudate in the airspaces. The alveolar walls are oedematous and thickened. This represents the histological correlate of acute exacerbation.
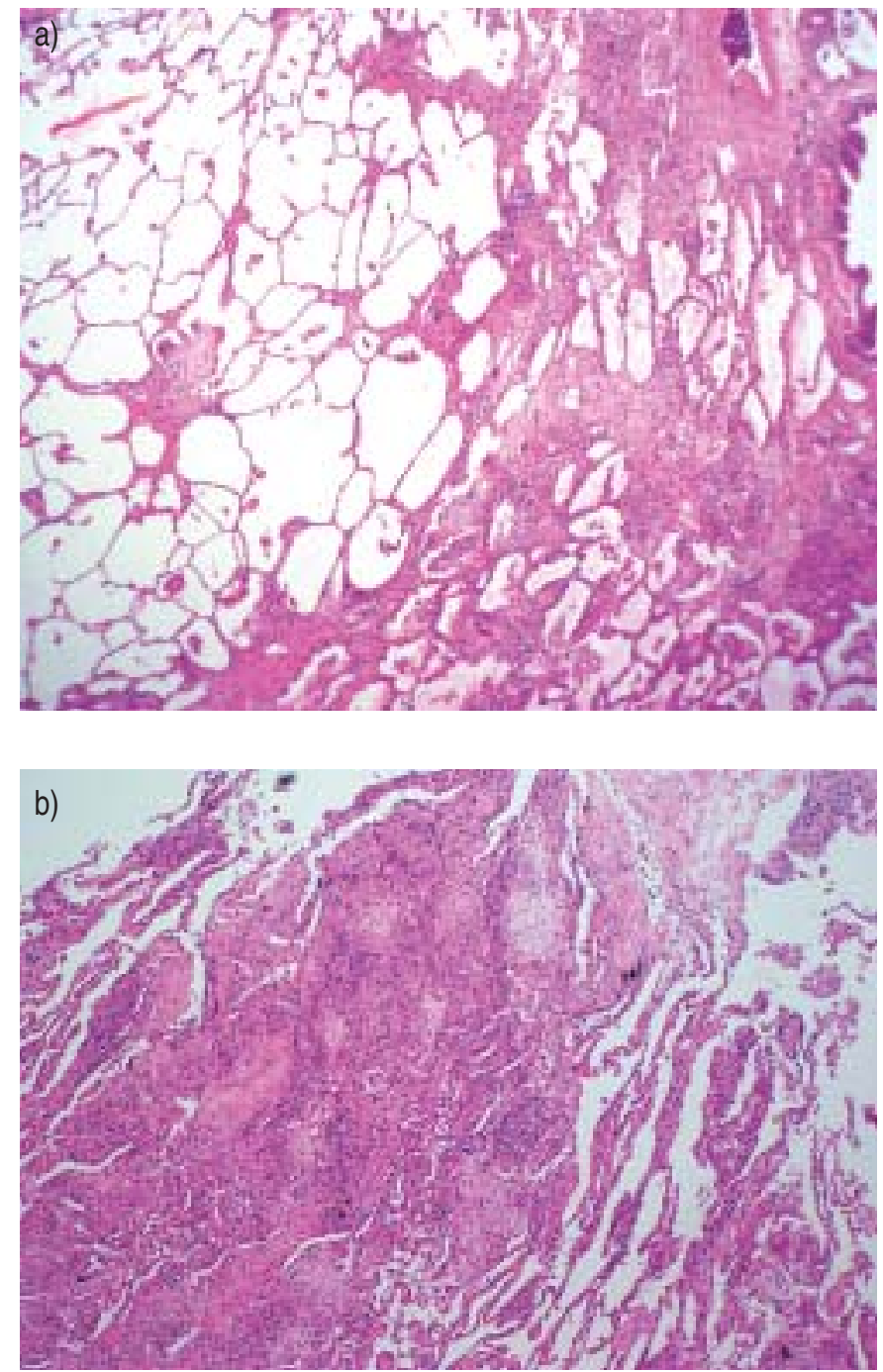

FIGURE 5. A 70-yr-old female presented with idiopathic pulmonary fibrosis and acute exacerbation. a) Typical background features of usual interstitial pneumonia with patchy dense fibrosis and architectural destruction (right half). b) The acute exacerbation in this case is manifested as a patch organising pneumonia involving the architecturally intact lung tissue and this field was identical to organising pneumonia as seen in bronchiolitis obliterans organising pneumonia/ cryptogenic organising pneumonia. 
have been several reports of AE after SLBx $[6,7]$, lung resection $[8,9]$, or BAL [10-12]. Furthermore, UTz et al. [29] reported that the short-term mortality rate $(16.7 \%)$ after SLBx was higher in patients with IPF/UIP, compared with the mortality rate of $1.7 \%$ in 771 unselected patients undergoing SLBx at the same institution [30]. In their report, the authors attributed $80 \%$ of the recorded deaths to acute lung injury. However, since those patients had already had IPF (because the subjects of that study were by definition patients with IPF), it is reasonable to think that these cases represented $\mathrm{AE}$ rather than acute lung injury of unknown aetiology. Indeed, when the authors reviewed the paper in greater detail, it was found that SLBx might have induced $\mathrm{AE}$ in $25 \%$ of their cases and the remaining $75 \%$ might have had $\mathrm{AE}$ at the time of biopsy. In the present study, two patients developed AE after SLBx and of the four patients biopsied after development of rapid aggravation, two patients had deteriorated further after the biopsy and died. Therefore, the findings of the present study are consistent with previous reports that SLBx can induce or aggravate the rapid worsening of respiratory conditions in IPF. Thus, from both the experience of the authors and the reports in the literature, it is now thought that it is both unnecessary and unethical to undertake SLBx at the time of rapid deterioration unless there are additional indications present, such as the exclusion of infection or associated malignancy.

Infection was the most important factor in the differential diagnosis of $\mathrm{AE}$, because many of the patients with IPF were treated with corticosteroids either with or without cytotoxic drugs. Although this study was retrospective, all the possible microbiological studies had been undertaken in all patients, other than those with procedure-related AE, in whom BAL was not performed because they were not immune-compromised at that time. All investigations were negative for causal organisms, excluding infection from the differential diagnosis. Gastro-oesophageal reflux may also have a bearing on the aetiology of $\mathrm{AE}$, as patients with IPF have significantly more oesophageal acid reflux than those with other interstitial lung diseases [31]. This needs to be assessed by further study.

HRCT revealed newly developed, widespread GGO superimposed on pre-existing subpleural reticular density with honeycombing, apart from the three patients who presented with AE without prior diagnosis. These patients' HRCTs showed honeycombing lesions in the subpleural area coexistent with widespread GGO and their surgical lung biopsies revealed UIP combined with DAD, indicating AE of IPF and excluding acute interstitial pneumonia.

Reports of treatment for AE are limited to small numbers of cases, although there are several papers reporting improvement with corticosteroid treatment $[14,17,28]$. TSUKAMOTO et al. [28] found that the age at onset of IPF, respiratory status before $\mathrm{AE}$ (carbon dioxide arterial tension), and inflammatory activity (C-reactive protein) were closely related to responsiveness and patient outcome. However, because all patients in this study were treated with corticosteroids and the mortality rate was so high, it was not possible to evaluate corticosteroid efficacy. Furthermore, no significant differences in the above parameters were found in this study's patients in relation to response to treatment or prognosis. However, there is a report that patients with multifocal and diffuse parenchymal opacification have a poorer prognosis than those with peripheral parenchymal opacification [18], and this study's results are consistent with this data.

In terms of the histology performed during $\mathrm{AE}$, the major histological finding was DAD, predominantly in the organising phase, as is well described in the pathology literature [32, 33]. While the appearances in a given microscopic field are identical to those seen in intra-alveolar organisation due to other causes such as COP, the presence of coexistent acute respiratory failure, and the HRCT data, plus the areas with the adjacent hyaline membranes in three of the four cases, indicate that the features represented organising DAD. However, although it has been suggested that a purely organising pattern is more favourable than a more acute pattern with hyaline membranes [18], the limitation of having only four patients precluded assessing this hypothesis. It was also not possible to identify any features within the background UIP that indicated predisposition to acute exacerbation.

In conclusion, this study shows that acute exacerbation in idiopathic pulmonary fibrosis has a frequency of $9.6 \%$ in 2 yrs. Data suggest that acute exacerbation can occur as a spectrum rather than a clearly definable event. The aetiology remains unknown. Further studies are required to assess the spectrum of such exacerbations, identify the risk factors, and determine the efficacy of treatment.

\section{REFERENCES}

1 Turner-Warwick MB, Burrows AJ. Cryptogenic fibrosing alveolitis: clinical features and their influence on survival Thorax 1980; 35: 171-180.

2 Selman M, King TE Jr, Pardo A. Idiopathic pulmonary fibrosis: prevailing and evolving hypotheses about its pathogenesis and implications for therapy. Ann Intern Med 2001; 134: 136-151.

3 Carrington CB, Gaensler EA, Coutu RE, Fitzgerald MX, Gupta RG. Natural history and untreated course of usual and desquamative interstitial pneumonia. $N$ Engl J Med 1978; 298: 801-809.

4 Katzenstein AL, Myers JL. Idiopathic pulmonary fibrosis. Clinical relevance of pathologic classification. Am J Respir Crit Care Med 1998; 157: 1301-1315.

5 American Thoracic Society. Idiopathic pulmonary fibrosis: diagnosis and treatment: international consensus statement. Am J Respir Crit Care Med 2000; 161: 646-664.

6 Tasaka S, Kanazawa M, Komune Y, et al. Acute exacerbation of subacute interstitial pneumonia after thoracoscopic lung biopsy. Nihon Kokyuki Gakkai Zasshi 1998; 36: 182-186.

7 Kanazawa M, Kawabata Y, Takayanagi N, et al. A questionnaire survey of surgical lung biopsy in patients with diffuse lung diseases. Nihon Kokyuki Gakkai Zasshi 2000; 38: 770-777.

8 Enomoto T, Kawamoto M, Kunugi S, et al. Clinicopathological analysis of patients with idiopathic pulmonary fibrosis which became acutely exacerbated after video-assisted thoracoscopic surgical lung biopsy. Nihon Kokyuki Gakkai Zasshi 2002; 40: 806-811.

9 Horio H, Nomori H, Morinaga S, Fuyuno G, Kobayashi R, Iga R. Exacerbation of idiopathic interstitial pneumonia 
after lobectomy for lung cancer. Nihon Kyobu Shikkan Gakkai Zasshi 1996; 34: 439-443.

10 Sugino S, Kanaya N, Namiki A. A case of acute exacerbation of idiopathic interstitial pneumonia after pneumonectomy. Masui 2000; 49: 187-190.

11 Suga T, Sugiyama Y, Ohno S, Kitamura S. Two cases of idiopathic interstitial pneumonia which developed acute exacerbation after bronchoalveolar lavage. Nihon Kyobu Shikkan Gakkai Zasshi 1994; 32: 174-178.

12 Hiwatari N, Shimura S, Takishima T, Shirato K. Bronchoalveolar lavage as a possible cause of acute exacerbation in idiopathic pulmonary fibrosis patients. Tohoku J Exp Med 1994; 174: 379-386.

13 Nishiyama O, Shimizu M, Ito $\mathrm{Y}$, et al. Effect of prolonged low-dose methylprednisolone therapy in acute exacerbation of idiopathic pulmonary fibrosis. Respir Care 2001; 46: 698-701.

14 Takahashi T, Munakata M, Ohtsuka $Y$, et al. Effects of corticosteroid pulse treatment on outcomes in acute exacerbations of idiopathic interstitial pneumonia. Nihon Kyobu Shikkan Gakkai Zasshi 1997; 35: 9-15.

15 Colby TV. Interstitial disease. In: Colby TV, Lombard CM, Yousem SA, Kitaichi M, eds. Atlas of Pulmonary Surgical Pathology. Philadelphia, WB Saunders, 1991; pp. 227-306.

16 Kitaichi M. Pathologic features and classification of interstitial pnueumonia of unknown etiology. Bull Chest Dis Res Inst Kyoto Univ 1990; 23: 1-18.

17 Kondoh Y, Taniguchi H, Kawabata Y, Yokoi T, Suzuki K, Takag K. Acute exacerbation in idiopathic pulmonary fibrosis. Analysis of clinical and pathologic findings in three cases. Chest 1993; 103: 1808-1812.

18 Akira M, Hamada H, Sakatani M, Kobayashi C, Nishioka M, Yamamoto S. CT findings during phase of accelerated deterioration in patients with idiopathic pulmonary fibrosis. AJR Am J Roentgenol 1997; 168: 79-83.

19 Rice AJ, Wells AU, Bouros D, et al. Terminal diffuse alveolar damage in relation to interstitial pneumonias. An autopsy study. Am J Clin Pathol 2003; 119: 709-714.

20 Ambrosini V, Cancellieri A, Chilosi M, et al. Acute exacerbation of idiopathic pulmonary fibrosis: report of a series. Eur Respir J 2003; 22: 821-826.

21 ATS/ERS. American Thoracic Society/European Respiratory Society international multidisciplinary consensus classification of the idiopathic interstitial pneumonias. Am J Respir Crit Care Med 2002; 165: 277-304.

22 Jegall YJ, Kim DS, Shim TS, et al. Physiology is a stronger predictor of survival than pathology in fibrotic interstitial pneumonia. Am J Respir Crit Care Med 2005; 171: 639-44.

23 Kim DS, Yong GJ, Tae SS, et al. The value of interleukin-12 as an activity marker of pulmonary sarcoidosis. Sarcoidosis Vasc Diffuse Lung Dis 2000; 17: 271-276.

24 Nicholson AG, Fulford LG, Colby TV, du Bois RM, Hansell DM, Wells AU. The frequency of fibroblastic foci in usual interstitial pneumonia and their relationship to disease progression. Am J Respir Crit Care Med 2002; 166: 173-177.

25 Raghu G. Idiopathic pulmonary fibrosis. A rational clinical approach. Chest 1987; 92: 148-154.

26 Panos RJ, Mortenson RL, Niccoli SA, King TE. Clinical deterioration in patients with idiopathic pulmonary fibrosis: causes and assessment. Am J Med 1990; 4: 396-404.

27 Kuwano K, Fukuyama S, Ikegami T, et al. An autopsy case of idiopathic interstitial pneumonia with diffuse alveolar hemorrhage due to acute exacerbation. Nihon Kokyuki Gakkai Zasshi 2001; 39: 787-791.

28 Tsukamoto K, Chida K, Hayakawa H, et al. Prediction of outcome after acute exacerbation of idiopathic interstitial pneumonia. Nihon Kyobu Shikkan Gakkai Zasshi 1997; 35: 746-754.

29 Utz JP, Ryu JH, Douglas WW, et al. High short-term mortality following lung biopsy for usual interstitial pneumonia. Eur Respir J 2001; 17: 175-179.

30 Allen MS, Deschamps C, Jones DM, Trastek VF, Pairolero PC. Video-assisted thoracic surgical procedures: the Mayo experience. Mayo Clinic Proc 1996; 71: 351-359.

31 Tobin RW, Pope II CE, Pellegrini CA, Emond MJ, Sillery J, Raghu G. Increased prevalence of gastroesophageal reflux in patients with idiopathic pulmonary fibrosis. Am J Respir Crit Care Med 1998; 158: 1804-1808.

32 Pratt PC. Pathology of adult respiratory distress syndrome. Monogr Pathol 1978; 19: 43-57.

33 Katzenstein AL. Acute lung injury patterns, Chapter 2. Katzenstein and Askin's Surgical Pathology of Nonneoplastic Lung Disease. 3rd Edn. Philadelphia, W.B. Saunders; 1997; pp: 14-47. 